\title{
ANÁLISIS DE LAS PREGUNTAS DOCENTES EN UN ENTORNO DE INDAGACIÓN SOBRE EL CICLO DEL AGUA
}

\author{
Ana Aragüés Díaz ${ }^{1}$ \\ Instituto de Educación Secundaria Valle del Ebro, Tudela \\ Departamento de Educación de Navarra
}

Recibido 14/11/2019 Aceptado 27/11/2019

\begin{abstract}
Este trabajo trata sobre un estudio de caso en el que una futura maestra lleva a cabo una actividad indagadora sobre el ciclo del agua en un aula de Primaria. El análisis se centra en examinar el tipo guía realizada por la futura maestra a través de las preguntas. Los resultados muestran cómo la planificación de una serie de preguntas sirve a la futura maestra como forma de control hacia una estructura a la que atenerse. Entre las dificultades encontradas destaca la falta de preguntas orientadas a establecer relaciones entre un modelo físico-químico de los cambios de estado.
\end{abstract}

$\checkmark$ This paper is about a case study in which a future teacher carries out an investigative activity on the water cycle in a Primary classroom. The analysis focuses on examining the type of guidance done by the future teacher through the questions. The results show how the planning of a series of questions serves the future teacher as a form of control towards a structure to be adhered to. Among the difficulties encountered, the lack of questions aimed at establishing relationships between a physical-chemical model of state changes stands out.

DOI

https://doi.org/10.15366/didacticas2019.21.003

PALABRAS CLAVE

Preguntas; Ciclo del agua; Primaria.

\section{KEYWORDS}

Questions; Water cycle; Primary. 


\section{INTRODUCCIÓN}

Actualmente en la literatura especializada existe un acuerdo en considerar el aprendizaje por indagación como una forma efectiva de construir conocimiento científico en la escuela (National Research Council, 2012; Otero y Crujeiras, 2016). Desde nuestra perspectiva, compartimos la idea de Vílchez y Bravo (2015) cuando señalan que la indagación hace referencia a una forma de aprendizaje en la que el docente plantea preguntas al alumnado haciendo de guía para la construcción de un conocimiento científico. De esta forma, indagar en el aula bien podría considerarse como una forma de aprender basada en preguntas (Crujeiras Pérez y Jiménez Aleixandre, 2015). En este proceso el rol del maestro se convierte en el de una guía capaz de proporcionar contextos y oportunidades para que los estudiantes adquieran determinados significados (Huffman y Kalnin, 2003). No obstante, y a pesar de los beneficios que parece aportar un aprendizaje de las ciencias por indagación (Linn et al. 2014), son varios autores los que señalan que se trata de una forma de enseñanza-aprendizaje poco extendida en las aulas (Gil Quílez et al., 2008). Entre los diversos motivos a los que se atribuye este hecho se encuentran aspectos relacionados con una falta de confianza del docente (Windschitl, 2003), posibles carencias en su marco teórico científico (Martin Dunlop, 2012) o quizá una comprensión poco clara de lo que implica un proceso de enseñanza-aprendizaje basado en la indagación.

Parece obvio deducir que si lo que se pretende es que los alumnos de Primaria indaguen deberán ser los futuros maestros los que adquieran, en primera instancia, una serie de habilidades basadas en el cuestionamiento, la reflexión, el razonamiento o el uso de pruebas (Cortés Gracia y de la Gándara Gómez, 2006). En este sentido, la etapa del Practicum universitario se plantea como una oportunidad clave para que futuros docentes exploren los retos que supone indagar con los alumnos (Brown, 2012).

Tradicionalmente la pregunta en la escuela ha tenido la misión de evaluar qué es lo que los estudiantes saben y lo que no. Con frecuencia la principal función de la pregunta en el aula no es más que la de obtener la 'respuesta correcta' en boca de los alumnos (Omairah, 2009). Desde una perspectiva de aprendizaje integral de las ciencias se apuesta por un aprendizaje no sólo basado en la reproducción y aplicación de conceptos sino también en la ejercitación de tareas propias científicas. Entre las propuestas didácticas más recomendadas para un adecuado aprendizaje de las ciencias destaca la indagación guiada (NRC, 2012). Esta perspectiva propone un aprendizaje que se centra tanto en el saber y como en el saber hacer, empleando para ello la metodología científica (Domènech Casal, 2014). Al igual que ocurre en la ciencia normal, la ciencia en la escuela debería ser el resultado de respuestas a una serie de preguntas. Windschitl (2003) plantea la idea de la indagación guiada como un proceso en el que el docente propone preguntas a sus estudiantes para debatir y explorar posibles soluciones con sus estudiantes. No obstante, resulta obvio que no todas las preguntas provocan por sí solas los razonamientos y/o estrategias de resolución esperadas (ej. observación, toma de datos, aplicación a otros contextos etc.). 
Investigaciones sobre las preguntas en el aula en relación a su relevancia, su adecuación o su sentido se concretan en diversas clasificaciones propuestas para las preguntas del docente (Brown, 2012; Chin, 2007; Koufetta-Menicou y Scaife, 2000; Marquéz Bargalló y Roca Tort, 2009). Estas clasificaciones han ido evolucionando a lo largo de los años a través de distintas perspectivas que abarcan desde trabajos fundamentados en una visión socrática (Richard, 1989) hasta aquellas centradas en la perspectiva del contexto del aula (Lemke, 1997). Son dos las principales perspectivas adoptadas para el estudio de las preguntas (Forero Sáenz, 2014). La primera, responde a un enfoque basado en un procesoproducto (Dillon, 1982). Los estudios realizados bajo esta perspectiva relacionan las preguntas de los docentes con los resultados de los estudiantes. Este tipo de estudios se enfocaban principalmente en relacionar preguntas de alto nivel cognitivo con las respuestas orales de los alumnos. Una de las críticas hacia este tipo de estudio fue que quizá que no era posible inferir que preguntas de alto nivel cognitivo promoviesen mayores aprendizajes que las de nivel bajo (Forero Sáenz, 2014). La segunda perspectiva, más reciente, para el estudio de las preguntas se basa en un enfoque sociolingüista. El lenguaje está directamente relacionado con la situación social (Carlsen, 1999) por lo que este tipo de estudios enfoca la atención en el análisis del discurso. En este sentido, la clasificación de las preguntas surge de forma deductiva al contexto (Lemke, 1997). El contenido pasa a estar relacionado con su contexto, siendo así necesario un exhaustivo análisis que tenga en cuenta las respuestas de los estudiantes para el análisis de las preguntas. Desde nuestra visión, examinar qué es lo que se ha dicho en el aula, el medio didáctico o las pruebas que los alumnos disponen son elementos imprescindibles para el estudio de las preguntas del profesor. De entre todos los sistemas de categorías propuestos en la literatura resulta especialmente útil el elaborado por Kawalkar y Vijapurkar (2013) en un entorno de indagación. Estas autoras, tras realizar un fino estudio sobre las transcripciones de varias lecciones en clases de Primaria, establecen cinco grandes categorías: Explorar requisitos previos, Generar ideas y explicaciones, Mayor sondeo de las respuestas iniciales, Refina conceptos e ideas de la primera explicación dada y Guiando a toda la clase hacia los conceptos científicos. La gradualidad de la demanda cognitiva es considerada de menor a mayor desde el primer grupo al último. Cabe mencionar cómo la relevancia del contexto otorgada en este tipo de estudios provoca que una misma pregunta pueda categorizarse en un grupo u otro. Es decir, la misma pregunta planteada al inicio o en la mitad de la lección podría ser asociada dentro del grupo Exploración de los requisitos previos o del grupo Refina conceptos e ideas (de la primera explicación dada), por ejemplo.

\section{Objetivo}

En el presente trabajo se examina una actividad que pretende ser de corte indagador sobre el ciclo del agua llevada a cabo por una futura maestra (etapa del Practicum) en un aula de 
Primaria ( $6^{\circ}$ curso, 11-12 años). En concreto la pregunta de investigación que rige el análisis es la siguiente: ¿Qué tipo de guía/estructura, a través de las preguntas, es realizada por la futura maestra durante la actividad?

\section{METODOLOGÍA}

\section{Contexto y participantes}

Este estudio se realiza dentro de un programa universitario de formación docente (Practicum) en el que una futura maestra trata de desarrollar una actividad basada en la indagación. Esta futura maestra trabajó durante su formación universitaria en entornos de indagación dentro de la asignatura de Didáctica del medio Biológico y Geológico. El programa se desarrolla en una escuela pública de España en una clase de un curso de $6^{\circ}$ de Primaria con un total de 17 estudiantes. El estudio se centra en el análisis de una actividad que surge a raíz de un debate anterior en el que los estudiantes estudiaban el modelo de ciclo del agua a través del clásico dibujo que aparece en los libros de texto. En este debate algunos estudiantes manifestaban que sólo llueve en las montañas mientras que otros defendían que también llueve en el mar. En este contexto, la futura maestra planificó una actividad con la intención de que los alumnos fuesen capaces de: a) transferir su modelo teórico de cambios de estado a un contexto experimental y b) emplear conocimientos sobre los de cambios de estado para hacer predicciones en otros contextos (en la atmósfera, en las montañas...).

Para la preparación de la actividad la futura maestra dio respuesta a las siguientes preguntas planteadas por su tutora de la Universidad: ¿Qué les voy a explicar?, ¿Cómo lo voy a llevar a cabo? y ¿Qué preguntas les voy a hacer a los alumnos? Las respuestas a las mismas dieron lugar a un sencillo experimento con dos vasos llenos con la misma cantidad de agua y cubiertos ambos con un film de plástico. Un día antes de realizar la sesión, uno de los vasos fue depositado al lado de la ventana cerca del radiador (vaso 1), mientras que el otro se situó en una zona oscura del aula sin una fuente calefacción cercana (vaso 2). Las preguntas que la futura maestra preparó se recogen en la Tabla 1.

\begin{tabular}{|l|}
\hline \multicolumn{1}{|c|}{ Preguntas planificadas (Ficha de los alumnos) } \\
\hline 1. ¿Qué ha pasado en los vasos? \\
\hline 2. ¿Dónde está el agua que había antes? \\
\hline 3. ¿Siempre hay la misma cantidad de agua? \\
\hline 4. ¿Por qué creéis que en un vaso hay más agua líquida que en otro? \\
\hline 5. ¿Sí pusiera un hielo encima del plástico, ¿qué pasaría? \\
\hline 6. ¿Pensáis que sólo llueve en las montañas? ¿Por qué? \\
\hline 7. ¿Cómo lo relacionaríais con este experimento? \\
\hline 8. Si pusiese los vasos igual, pero de noche ¿pasaría lo mismo? \\
\hline
\end{tabular}

Tabla 1. Preguntas planificadas 
Al comienzo de la sesión la futura maestra entregó una ficha a cada alumno con las preguntas. Los alumnos se dispusieron a observar los siguientes hechos: a) la aparición de gotas en el film de plástico en el vaso 1 cerca de la ventana y del radiador (dato 1) y b) la no aparición de gotas en el vaso 2 situado en el otro extremo del aula (dato 2). Cabe señalar que el alumnado no había trabajado previamente aspectos del modelo cinético-molecular sobre los cambios de estado.

\section{Instrumento}

El enfoque metodológico se basa en una investigación de tipo cualitativo de estudio de caso. La fuente de datos de la que se dispone es una grabación de video y audio de 1 hora. Para el análisis se ha empleado la herramienta Transana (Enlace). En este apartado, queremos destacar la importancia sobre el uso de las grabaciones de audio y video si lo que se pretende es conocer qué se hace y qué se dice en el aula. Este tipo de registros permiten capturar toda la complejidad del aula ofreciendo datos que van más allá del momento estático que ofrecen los registros escritos o los cuestionarios (Tiberghien y Sensevy, 2012). El video muestra la ventaja de proporcionar registros orales, gestuales y escritos, como por ejemplo un dibujo en la pizarra, los cuales son imprescindibles para una adecuada comprensión del contexto del aula.

\section{ANÁLISIS}

\section{Análisis de la estructura de la actividad: los juegos didácticos}

Desde la Teoría de Acción Conjunta en Didáctica (TADC; Sensevy, 2011), el aula se considera como un sistema complejo que puede describirse en términos de juegos didácticos. De forma similar a una obra de teatro, la clase se estructura en distintas escenas. Cada juego didáctico puede considerarse una escena que tiene una coherencia temática y un objetivo o regla común (Sensevy, 2011). Se considera que un nuevo juego didáctico aparece cuando se detecta un cambio en el contrato didáctico establecido (reglas, generalmente explícitas de forma oral) o del contexto del aula (cambios en el medio didáctico, aparición de nuevos elementos materiales o modificación de la organización del aula). El análisis en términos de secuencia de juegos didácticos nos ofrece una visión general de lo que sucede en la clase.

La Tabla 2 resume la secuencia de los 17 juegos en los que se ha organizado la actividad. Después de visualizar la video-grabación varias veces con las transcripciones, identificamos los juegos didácticos, asignando a cada uno un título y duración (Tabla 2). 


\begin{tabular}{|l|c|}
\hline \multicolumn{1}{|c|}{ Juegos didácticos/Título } & Tiempo \\
\hline J1. ¿En qué va a consistir el experimento? & $(0: 00: 05-0: 01: 39)$ \\
\hline J2. ¿Qué ha pasado en el vaso? & $(0: 01: 39-0: 02: 31)$ \\
\hline J3. ¿Por qué hay gotitas en el plástico? & $(0: 02: 31-0: 04: 52)$ \\
\hline J4. ¿Hay la misma cantidad de agua? & $(0: 04: 52-0: 06: 27)$ \\
\hline J5. ¿Y si ponemos un hielo encima del plástico? & $(0: 06: 27-0: 12: 41)$ \\
\hline J6. ¿Sólo llueve en las montañas? & $(0: 19: 41-0: 19: 40)$ \\
\hline J7. ¿Por la noche ocurrirá lo mismo? & $(0: 22: 37-0: 26: 49)$ \\
\hline J8. ¿Qué ha pasado en el vaso? & $(0: 26: 49-0: 29: 12)$ \\
\hline J9. ¿Qué pasa si pones un hielo en el plástico? & $(0: 29: 12-0: 33: 38)$ \\
\hline J10. ¿Por qué llueve? & $(0: 33: 38-0: 35: 11)$ \\
\hline J11. ¿Dónde está el agua que había antes? & $(0: 35: 11-0: 36: 37)$ \\
\hline J12. ¿Por qué hay en un vaso más agua líquida que en el otro? & $(0: 36: 37-0: 38: 07)$ \\
\hline J13. ¿Sólo llueve en las montañas? & $(0: 38: 07-0: 50: 39)$ \\
\hline J14. ¿Cómo lo relacionarías con el experimento (lluvia)? & $(0: 50: 39-0: 55: 57)$ \\
\hline J15. ¿Por qué a veces llueve o nieva? & $(0: 55: 57-0: 56: 28)$ \\
\hline J16. ¿Siempre hay la misma cantidad de agua en las nubes? & $(0: 56: 28-1: 00: 03)$ \\
\hline J17. ¿Pasaría lo mismo por la noche? & \\
\hline
\end{tabular}

Tabla 2. Actividad estructurada en juegos didácticos

\section{Categorización de las preguntas docentes}

Para el análisis de las preguntas planteadas por la futura docente se ha realizado un sistema de categorías basado en el trabajo de Kawalkar y Vijapurkar (2013) habiéndose añadido dos categorías más: Extender aplicabilidad y Otras. (Tabla 3)

\begin{tabular}{|l|l|}
\hline \multicolumn{1}{|c|}{ Niveles de demandas cognitivas de las preguntas docentes } & \multicolumn{1}{|c|}{ Ejemplos } \\
\hline $\begin{array}{l}\text { Q1. Explorar requisitos previos (recuerdo de los hechos, de lo que fue dicho, } \\
\text { observado, experiencias previas, medir la comprensión de los requisitos pre- } \\
\text { vios). }\end{array}$ & $\begin{array}{l}\text { ¿Para qué es este ex- } \\
\text { perimento? }\end{array}$ \\
\hline $\begin{array}{l}\text { Q2. Generar ideas y explicaciones (dirigir la atención, preguntar para explicar, } \\
\text { para guiar el razonamiento, preguntar por una opinión, alentar una mayor res- } \\
\text { puesta). }\end{array}$ & $\begin{array}{c}\text { ¿Por qué el agua se ha } \\
\text { evaporado? }\end{array}$ \\
\hline $\begin{array}{l}\text { Q3. Mayor sondeo de las respuestas iniciales (clarificatoria, solicita elabora- } \\
\text { ción, señala contradicciones, da a entender, refrasea respuestas de los estu- } \\
\text { diantes ). }\end{array}$ & $\begin{array}{l}\text { Pero ¿qué ocurre con } \\
\text { el agua ahí dentro? }\end{array}$ \\
\hline $\begin{array}{l}\text { Q4. Refinar conceptos e ideas (pregunta para probar o averiguar, solicita infe- } \\
\text { rencia, ayuda a realizar conexiones, aspectos no considerados, señala fallos } \\
\text { en el argumento, conduce al punto central, invoca un pensamiento reflexivo). }\end{array}$ & $\begin{array}{l}\text { Y ¿por qué las gotas } \\
\text { están cayendo? }\end{array}$ \\
\hline $\begin{array}{l}\text { Q5. Guiando a toda la clase hacia los conceptos científicos (hace un balance, } \\
\text { considera una evaluación del conocimiento, insta a considerar una variedad de } \\
\text { puntos de vista, asociación a terminología científica. }\end{array}$ & $\begin{array}{l}\text { Un momento, ¿cómo } \\
\text { hemos llamado a } \\
\text { esto? }\end{array}$ \\
\hline $\begin{array}{l}\text { Q6. Extender aplicabilidad (a través de la generalización, capacidad para pre- } \\
\text { decir, propone hipótesis o conjeturas). }\end{array}$ & $\begin{array}{l}\text { ¿Qué ocurre cuando la } \\
\text { temperatura cambia? }\end{array}$ \\
\hline $\begin{array}{l}\text { Q7. Otras (no relevantes o relacionadas con aspectos no relativos al tema de } \\
\text { estudio). }\end{array}$ & $\begin{array}{l}\text { Perdona iqué era lo } \\
\text { que me preguntabas? }\end{array}$ \\
\hline
\end{tabular}

Tabla 3. Sistema de categorías para las preguntas del maestro en entornos de indagación. 
El grupo Extender aplicabilidad es creado desde la idea planteada por Omariah (2009) cuando señala que un conocimiento no es realmente aprendido hasta que el estudiante no es capaz de aplicarlo a un contexto distinto del que ha sido creado. Además uno de los objetivos de la actividad era que los alumnos fuesen capaces de trasladar los conocimientos de un modelo físico-químico a un contexto hidro-geológico, de ahí su pertinencia. Por último, también se ha añadido el grupo de relativo a Otras con el objetivo de poder identificar todas aquellas preguntas que no guardan relación con el contenido que se pretende trabajar.

\section{Codificación y validación}

Para la codificación se procedió al escrutinio de las transcripciones así como al visionado sucesivo de la video-grabación. En la medida que el investigador interpreta que un enunciado hace referencia a una pregunta se procedió a la codificación mediante un código asignado. La codificación fue validada mediante triangulación (con un acuerdo del 90\%).

\section{RESULTADOS}

\section{Estructuración de la sesión: Juegos didácticos}

La actividad se estructura en 17 juegos didácticos (ver apartado de Metodología y análisis) regidos todos ellos por una de las preguntas que la futura maestra planificó que cada alumno posee de manera que los objetivos de la actividad son conocidos por todos los estudiantes.

La lección se estructura en dos partes claramente diferenciadas. Durante la primera parte (del juego 1 al 7), la futura maestra introduce una a una las preguntas recogidas en la ficha ofreciendo un debate sobre ellas a los estudiantes. En la segunda parte (del juego 8 al 17) los juegos didácticos que son planteados se orientan de nuevo a dar respuesta a las mismas preguntas. Pudiera parecer que existe una repetición de juegos didácticos durante la actividad, sin embargo debemos tener en cuenta que el contexto didáctico es claramente distinto y por tanto la función didáctica también lo es. Las preguntas planificadas por la futura maestra (Tabla 1) le sirven como forma de andamiaje para la estructuración de la actividad. Por otro lado, cabe resaltar cómo dos juegos didácticos (juegos 9 y 10), regidos por las preguntas ¿por qué a veces llueve o nieva? y ¿siempre hay la misma cantidad de agua en las nubes?, son introducidos por dos alumnas.

\section{Las preguntas espontáneas de la futura maestra}

Como forma de avanzar en el conocimiento durante la lección la futura maestra ejerce una guía fundamentada en un cuestionamiento constante en cada uno de los juegos didácticos 
(Figura 1). Además, como forma de involucrar a los alumnos en nuevos razonamientos suele utilizar sus respuestas para que éstos se vean obligados a replantear sus argumentos.

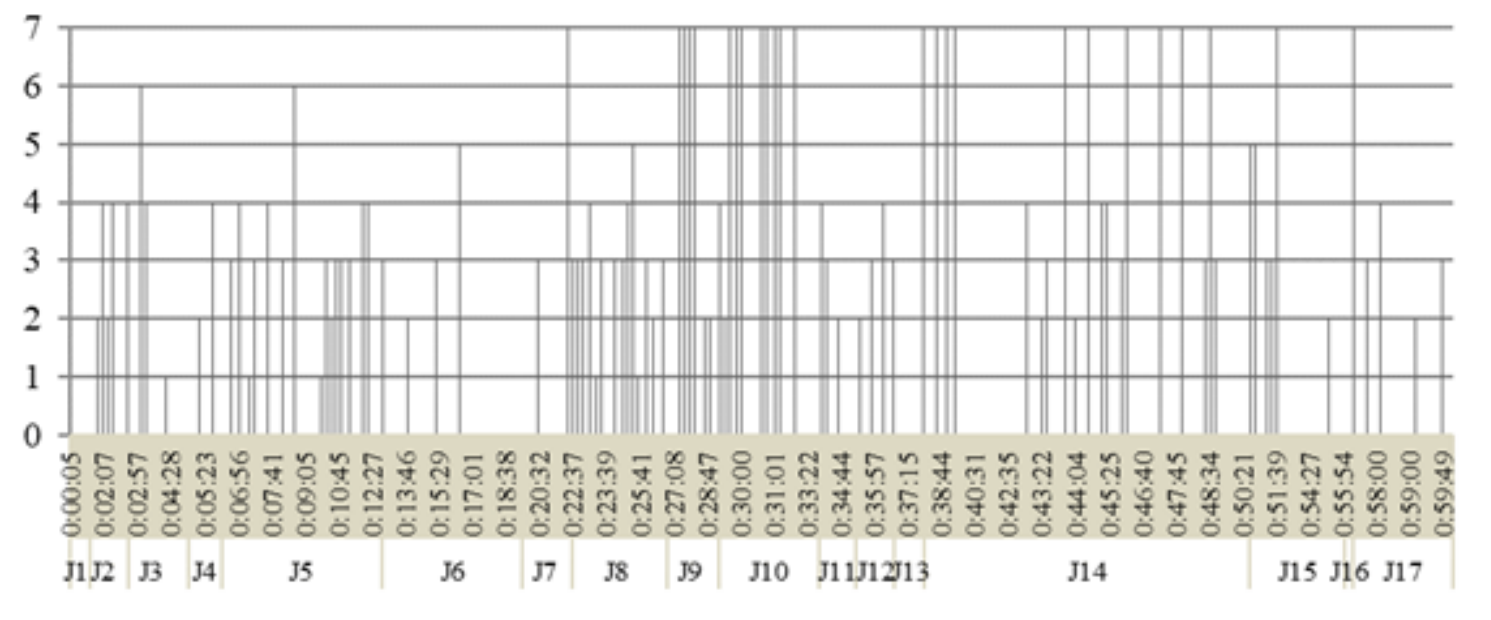

Figura 1. Emergencia de las preguntas durante la actividad. Leyenda:

Eje y: tipo de pregunta, (1)=Explorar pre-requisitos/preparar el terreno, (2)=Generar ideas y explicaciones, (3) = Mayor sondeo -de las respuestas iniciales-, (4)= Refina conceptos e ideas -de la primera explicación-,

(5) = Guiando a toda la clase hacia los conceptos científicos, (6)= Extender aplicabilidad, (7)=0tras

$$
\text { Eje } \mathrm{x} \text { : Jx, J=Juego, } \mathrm{x}=1,2, \ldots 7
$$

Como forma de avanzar en el conocimiento durante la lección la futura maestra ejerce una guía fundamentada en un cuestionamiento constante en cada uno de los juegos didácticos (Figura 1). Además, como forma de involucrar a los alumnos en nuevos razonamientos suele utilizar sus respuestas para que éstos se vean obligados a replantear sus argumentos.

Estudiante: Yo creo que no, ¿no?, porque desde aquí, algunas veces hace frío y otras veces hace calor, entonces ahí también puede variar la temperatura.

Futura maestra: Claro que puede variar, ¿y por qué cambia aquí la temperatura?

Estudiante: Por la posición del Sol.

Futura maestra: Vale, una cosa es por la posición del Sol, ¿por qué más cambia aquí la temperatura?

El análisis muestra también cómo las preguntas planteadas a los estudiantes les instan a considerar los datos aportados por el sencillo experimento.

Futura maestra: Entonces eso lo sabéis, que cuando hace calor se evapora. Bueno, se ha quedado ahí porque está el plástico, pero ¿por qué hay gotitas y no se queda solo gas? 
Si atendemos a cada uno de los 17 juegos didácticos observamos que no se aprecian claras evoluciones lineales en cuanto a las demandas cognitivas de las preguntas. Este dato se relaciona con el hecho de que la futura maestra tiene en cuenta las respuestas no solo de un único estudiante sino de todos ellos; y esta dinámica le obliga a plantear de nuevo preguntas con menor demanda cognitiva cuando no considera adecuadas ciertas respuestas.

Futura maestra: ¿Cuándo se evaporan cómo están? (Q3)

Estudiante 1: En estado gaseoso.

Futura maestra: Si, pero ¿y de temperatura? (Q4)

Estudiante 2: Normal.

Futura maestra: Para que ¿se evapore el agua? (Q4)

Estudiante 3: Ah, caliente.

Futura maestra: Entonces habéis dicho que las gotas se forman por el contraste, ¿si pongo un hielo es contraste? (Q2)

Para una visión porcentual de cada tipo de pregunta se ha elaborado la Figura 2:

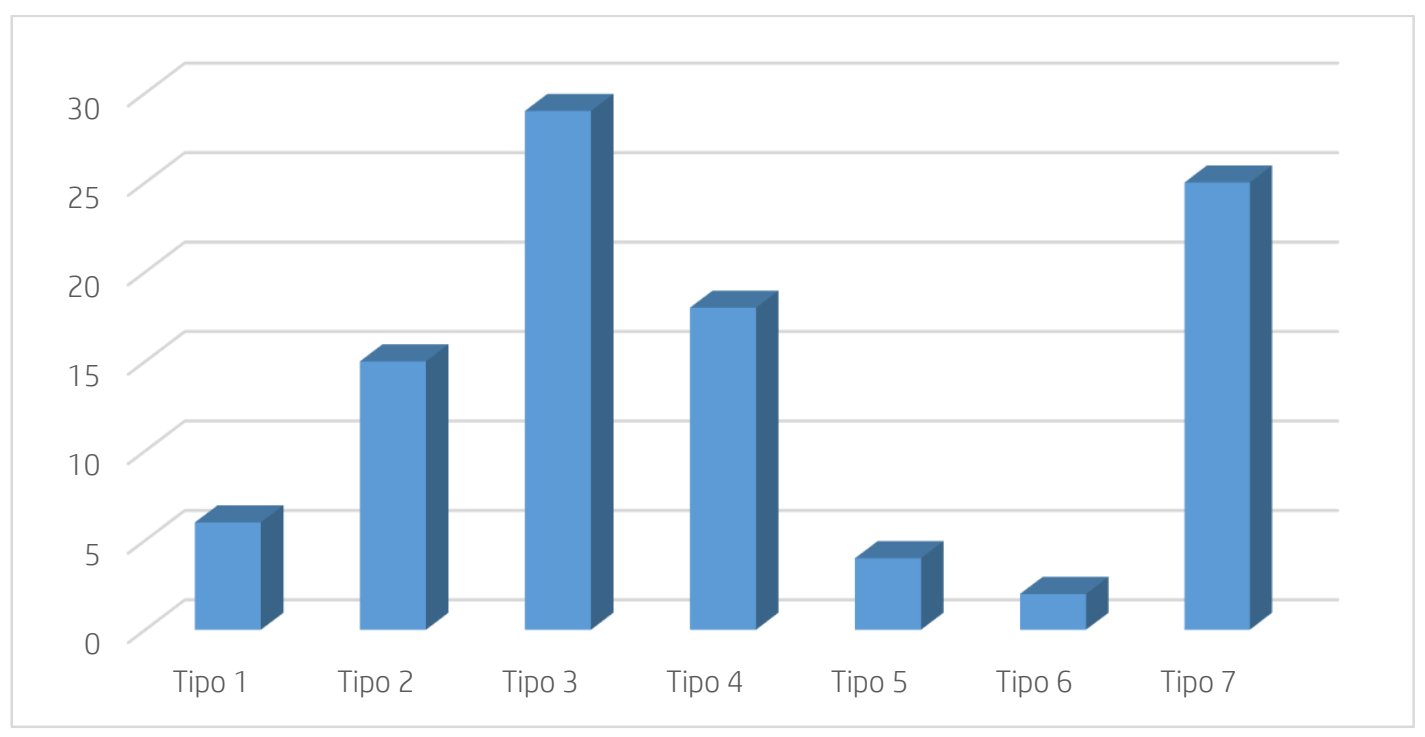

Figura 2. Porcentajes del tipo de preguntas. Leyenda:

Eje y: \% del tipo de pregunta

Eje $x$ : tipo de pregunta, $x=(1)=$ Explorar pre-requisitos/preparar el terreno, (2)=Generar ideas y explicaciones, (3)= Mayor sondeo - de las respuestas iniciales,$-(4)=$ Refina conceptos e ideas - de la primera explicación-, (5)= Guiando a toda la clase hacia los conceptos científicos, (6)= Extender aplicabilidad, (7)=0tras 
Al margen del porcentaje asociado a las preguntas del grupo Otras (Q7), la Figura 2 ofrece una distribución de 'u' invertida cuyo pico máximo corresponde a preguntas tipo Mayor sondeo -de las respuestas iniciales- (Q3) con un 29\%. Esto se revela como un esfuerzo constante por parte de la futura maestra para que el alumnado reconsidere sus primaras explicaciones. Cuando los alumnos parecen encontrar dificultades en conectar sus explicaciones, la futura maestra les guía planteando preguntas de tipo Q3 con la intención de ayudarles a alcanzar al objetivo del juego. La presencia de preguntas tipo Refina conceptos e ideas -de la primera explicación- (Q4) con un 18\% (Figura 1) se concentra entre los juegos 1 y 5 (Figura 2). Esto sugiere que por algún motivo durante la segunda mitad de la actividad la futura maestra parece no solicitar inferencias u orientar a realizar nuevas conexiones sobre aspectos que los estudiantes no hayan contemplado. En cuanto a las preguntas de mayor demanda cognitiva, Guiando a toda la clase hacia los conceptos científi$\cos ($ Q5) y Extender aplicabilidad (Q6), ambas poseen porcentajes relativamente bajos (4\% y $6 \%$ respectivamente, Figura 2). Es posible que la inexperiencia de esta futura docente sumado al hecho de tratar de aplicar una metodología de corte indagatorio en el aula, provoque que durante el juego 9 pierda, en cierta medida, el control del aula. Durante este juego el discurso se traslada a aspectos no relacionados con el objeto de estudio (ej. tifones, tormentas en el mar etc.). Con el fin de reconducir a los estudiantes hacia a los objetivos propuestos la futura maestra plantea nuevas preguntas tomando las respuestas de los estudiantes, lo que provoca un gran número de preguntas asociadas al grupo Otras (Q7) (25\%, Figura 2), que se extiende además en más juegos didácticos.

Los datos muestran una actividad caracterizada por un constante planteamiento de preguntas por parte de la futura maestra. La alternancia entre preguntas de alta y baja demanda cognitiva (Figura 1) es consecuencia de la adaptación de la futura maestra a las distintas respuestas de cada estudiante, las cuales le obligan a re-adaptar constantemente su demanda. En este sentido, se puede apreciar que hacer ciencia en la escuela implica hacer ciencia para todos, en la medida de que todas las respuestas por peregrinas que parezcan, han de ser tomadas en cuenta para avanzar en el aprendizaje.

En la primera parte de la actividad (hasta el juego didáctico 7) los resultados muestran un gran número de preguntas que instan a los alumnos a mejorar sus respuestas iniciales. Ello parece provocar la emergencia de un gran número de facetas que tratan de aportar explicaciones sobre los cambios de estado (Figuras 3 y 4). En contraposición, durante la segunda parte (del juego 8 al 17) parece suceder un aparente estancamiento en la aparición de facetas (Figura 3). Es en esta segunda parte cuando la futura maestra muestra dificultades a la hora de reconducir el diálogo a los objetivos de la sesión. Al tratar de emplear las respuestas de los estudiantes para plantear nuevas preguntas termina por alejarse de los contenidos que pretendía trabajar, trasladándose el debate a fenómenos como por ejemplo, qué son los tifones. Ello justifica el elevado número de preguntas tipo Q7. Otras que podemos encontrar durante la segunda parte de la actividad. 
Ante esta aparente dificultad de los alumnos para integrar los modelos de cambio de estado dentro del ciclo del agua hubiera sido deseable que la futura maestra hubiese planteado un mayor número de cuestiones tipo Q5. Guiando a toda la clase hacia los conceptos científicos, así como Q6. Extender aplicabilidad.

\section{CONCLUSIONES}

Este trabajo trata contribuir a los estudios de investigación centrados en el análisis de las prácticas docentes previas al ejercicio docente. La metodología de análisis empleada para la caracterización del aula a través de juegos didácticos (TACD; Sensevy, 2011), así como el estudio del discurso de la docente y de los alumnos, a través del tipo de preguntas y respuestas, ha permitido una fina descripción de lo que acontece cuando se trata de llevar a cabo una actividad de indagación.

La previa preparación de la actividad basada en la reflexión facilitó a la futura maestra una mayor seguridad en la gestión del aula. En otras palabras, la futura docente se enfrentó a la actividad desde una estructuración previa, basada en preguntas, a la que atenerse, salvando así la sensación de improvisación que la indagación plantea. Además, durante la segunda parte de la actividad, la futura maestra fue capaz de ceder la responsabilidad del aprendizaje a los estudiantes, en la medida que dos preguntas fueron planteadas por dos alumnas (¿Por qué a veces llueve o nieva? y ¿Siempre hay la misma cantidad de agua en las nubes? desencadenan dos pequeños debates, hecho considerado muy positivo desde el punto de vista de la indagación (NRC, 2012).

El tipo de guía ejercida por esta futura docente se orientó en un constante planteamiento de preguntas que trataban de adecuarse a las respuestas ofrecidas por el alumnado. La gran mayoría de preguntas son del tipo Mayor sondeo -de las respuestas iniciales-. Esto se debe en parte a que la futura docente no se conforma con una única respuesta, sino que toma en cuenta todas las respuestas del alumnado. Concluimos que hacer indagación en un aula de Primaria implica, a diferencia de otras etapas superiores, tener en cuenta todas las ideas del alumnado. Otro dato que es apreciado es la constante alternancia en cuanto a las demandas cognitivas de las preguntas. Al tener en cuenta cada una de las respuestas ella se ve constantemente obligada a readaptar el tipo de demanda. De esta forma, parece ser que la visión de la indagación que la futura docente presenta se encuentra muy relacionada con la idea de dar voz al alumnado.

En cuanto a las dificultades encontradas destaca la pérdida de control sobre el aula que la futura docente experimenta cuando trata de utilizar cada respuesta del alumnado para plantear una nueva pregunta. Esta estrategia le lleva a temas que se alejan del objeto de estudio como por ejemplo cuando los alumnos acaban hablando sobre los tifones en el mar. También se aprecia una falta de preguntas de alto nivel cognitivo (Guiando a toda la clase hacia los conceptos científicos o Extender aplicabilidad), especialmente en los casos en los que las respuestas del alumnado explicitan algún tipo de idea alternativa. En este sentido, 
interpretamos que la futura docente otorga más importancia a que cada alumno manifieste sus ideas que a definir con mayor precisión los conceptos. Algunas de las ideas alternativas expresadas por el alumnado (ej. que la evaporación se origina por el Sol, de manera que si está nublado no se origina evaporación, o que las nubes están formadas por sólo gas) surgen a raíz de preguntas que instan transferir un modelo de cambios de estado a un contexto hidro-geológico. Se encuentra así la dificultad que el alumnado posee a la hora de movilizar estas ideas alternativas clásicas sobre el ciclo del agua (Márquez, Izquierdo y Espinet, 2003), sólo siendo son capaces de reconsiderarlas cuando se les enfrenta a contextos contradictorios frente a sus explicaciones.

Es importante recordar, que para el estudio del ciclo del agua en el aula solo es posible reproducir aspectos parciales del proceso como son la evaporación o la condensación, lo que conlleva a que los docentes planteen razonamientos basados en datos de naturaleza teórica y no en evidencias. En este sentido, es importante incidir en la elaboración de actividades basadas en preguntas orientadas a que los estudiantes transfieran contenidos (modelo de cambios de estado) a otros contextos distintos (modelo hidro-geológico), facilitando así una adquisición real del conocimiento.

Para finalizar, queremos enfatizar cómo la actividad presentada, basada en un sencillo experimento, permitió desarrollar todo un proceso de razonamiento y cuestionamiento con el alumnado (Chin, 2007). Con este estudio queremos destacar el contexto del Practicum como momento integrador de teoría y práctica docente. Se trata de una oportunidad en la que sería conveniente crear un espacio para la reflexión sobre lo que un docente pretende hacer en clase y lo que realmente hace. Para concluir, señalar que este análisis nos ha permitido comprender algunas limitaciones experimentadas por una futura maestra durante la implementación de una actividad de indagación, se espera que los resultados obtenidos sean útiles para la práctica de otros futuros maestros.

\section{REFERENCIAS}

BROUSSEAU, G. (2006): "Theory of didactical situations in mathematics: Didactique des mathématiques”: Springer Science \& Business Media, 19, pp. 1970-1990.

BROWN, K. B. (2012). "Seeking Questions, Not Answers: The Potential of Inquiry-Based Approaches to Teaching Library and Information Science”. Journal of Education for Library and Information Science, 53 (3), pp. 189-199.

CHIN, C. (2007). "Teacher Questioning in Science Classrooms: Approaches that Stimulate Productive Thinking”. Journal of Research in Science Teaching, 44 (6), pp. 815-843. 
CRUJEIRAS PÉREZ, B. y JIMÉNEZ ALEIXANDRE, M. P. (2015). “Desafíos planteados por las actividades abiertas de indagación en el laboratorio: articulación de conocimientos teóricos y prácticos en las prácticas científicas”. Enseñanza de las ciencias, 33 (1), pp. 63-84.

DILLON, J. T. (1982). "The effect of questions in education and other enterprises". Journal of Curriculum Studies, 14 (2), pp. 127-152.

DOMĖNECH CASAL, J. (2014). "Una secuencia didáctica de modelización, indagación y creación del conocimiento científico en torno a la deriva continental y la tectónica de placas”. Revista Eureka sobre Enseñanza y Divulgación de las Ciencias, 12 (1), pp. 186197.

FORERO SÁENZ, AMPARO (2014). "El uso de las preguntas por parte del docente en la clase de matemáticas y sus efectos en las respuestas y conversaciones de los niños (Tesis Doctoral)”. Universidad Autónoma de Barcelona, Barcelona, España.

GIL, M. J., MARTÍNEZ, B., DE LA GÁNDARA, M., CALVO, J. M. y CORTÉS, A. L. (2008). "De la universidad a la escuela: no es fácil la indagación científica". Revista Interuniversitaria de Formación del Profesorado, 63 (22), pp. 81-100.

HUFFMAN, D., \& KALNIN, J. (2003). "Collaborative inquiry to make data-based decisions in schools”. Teaching and teacher education, 19 (6), pp. 569-580.

KAWALKAR, A., \& VIJAPURKAR J. (2013). "Scaffolding science talk: The role of teachers questions in the inquiry classroom”. International Journal of Science Education, 35 (12), pp. 2004-2027.

KOUFETTA-MENICOU, C., \& SCAIFE, J. (2000). "Teachers' questions-types and significance in science education”. School Science Review, 81, pp. 79-84.

LEMKE, J.L. (1997). Aprender a hablar ciencia. Lenguaje, aprendizaje y valores. Barcelona: Paidós.

LINN, M. C., GERARD, L., RYOO, K., MCELHANEY, K., LIU, O. L., \& RAFFERTY, A. N. (2014). "Computer-guided inquiry to improve science learning”, Science, 344 (6180), pp. 155-156.

MARQUÉZ BARGALLÓ C. M., y ROCA TORT, M. (2009). "Plantear preguntas: un punto de partida para aprender ciencias”. Revista Educación y pedagogía, 18 (45), pp. 61-71.

MÁRQUEZ C., IZQUIERDO, M. y ESPINET M. (2003). "Comunicación multimodal en la clase de ciencias: el ciclo del agua”, Enseñanza de las Ciencias, 21 (3), pp. 371-386.

MARTIN-DUNLOP, C. S. (2013). "Prospective elementary teachers' understanding of the nature of science and perceptions of the classroom learning environment", Research in Science Education, 43 (3), pp.873-893. 
NATIONAL RESEARCH COUNCIL. (2012). A framework for K-12 science education: Practices, crosscutting concepts, and core ideas. National Academies Press.

OMARIAH, O. (2009). "Teachers' Questioning Techniques and Their Potential in Heightening Pupils' Inquiry”. International Conference on Primary Education. Proceedings.

OTERO, S. y CRUJEIRAS, B. (2016). "Indagación en el laboratorio de física de secundaria: ¿cuáles serían las mejores condiciones para hacer kayak?". ReiDoCrea, 5, pp. 235-246.

RICHARD. W. P. (1989). "Critical thinking in North America: A new theory of knowledge, learning, and literacy”, Argumentation, 3 (2), pp. 197-235.

SENSEVY, G. (2011). Le sens du savoir. Éléments pour une théorie de l'action conjointe en didactique. Bruxelles: de Boeck.

TIBERGHIEN, A., \& SENSEVY, G. (2012). The nature of video studies in science education. In Science education research and practice in Europe, pp. 141-179. Sense Publishers, Rotterdam.

VÍLCHEZ GONZÁLEZ, J. M. y BRAVO TORIJA, B. (2015). "Percepción del profesorado de ciencias de educación primaria en formación acerca de las etapas y acciones necesarias para realizar una indagación escolar”. Enseñanza de las Ciencias, 33 (1), pp. 185202.

WINDSCHITL, M. (2003). "Inquiry projects in science teacher education: What can investigative experiences reveal about teacher thinking and eventual classroom practice?". Science Education, 87 (1), pp. 112-143. 